\title{
Hybrid Repair of Ascending Aortic Pseudoaneurysm
}

David Blitzer ${ }^{1}$, Stephanie Nguyen ${ }^{1}$, Virendra I. Patel ${ }^{2}$, Isaac George ${ }^{3}$, and Hiroo Takayama $^{3}$

${ }^{1}$ New York-Presbyterian/Columbia University Medical Center

${ }^{2}$ Columbia University Vagelos College of Physicians and Surgeons

${ }^{3}$ Columbia University College of Physicians and Surgeons

November 18, 2020

\begin{abstract}
For ascending aortic pseudoaneurysms with a large aortic entry site and thrombus burden, temporary stent-graft placement at induction of circulatory arrest can prevent thrombotic complication.
\end{abstract}

\section{Hybrid Repair of Ascending Aortic Pseudoaneurysm}

David Blitzer, MD ${ }^{1}$, Stephanie N. Nguyen, MD¹, Virendra Patel, MD, Isaac George, MD ${ }^{1}$, Hiroo Takayama, $\mathrm{MD}^{1}$

Columbia University, Department of Surgery, New York, NY, United States

Disclosures/Conflicts of Interest: None

Funding: None

Data Availability : Data sharing not applicable to this article as no datasets were generated or analysed during the current study

Clinical Trial Registration : Not Applicable

Word Count: 954

Corresponding Author:

Hiroo Takayama, MD, PhD

Division of Cardiac, Thoracic, and Vascular Surgery

Columbia University Medical Center

177 Fort Washington Ave

New York, NY 10019

Email:ht2225@cumc.columbia.edu

Abstract: For ascending aortic pseudoaneurysms with a large aortic entry site and thrombus burden, temporary stent-graft placement at induction of circulatory arrest can prevent thrombotic complication.

Ascending aortic pseudoaneurysm (PSA) is a rare, yet highly morbid complication of cardiac surgery, predisposing to aortic rupture, thrombosis, or distal embolization.[1,2] Due to high rates of morbidity and mortality for surgical PSA repair, transcatheter approaches have also been proposed for select patients with 
a narrow PSA 'neck' and sufficient landing zone.1-4 In this report, we describe successful application of a hybrid technique employing a thoracic endovascular aortic repair (TEVAR) to prevent embolic complications due to PSA thrombus migration during surgical PSA repair.

\section{Clinical Summary}

IRB approval, a consent statement, and clinical trial registration are not applicable for this study. A 53year-old male underwent coronary artery bypass grafting with a postoperative course complicated by MRSA mediastinitis, requiring multiple mediastinal re-explorations and an eventual flap closure. He was readmitted 2 months later with CTA chest demonstrating a 3.6-cm bilobed PSA with a large entry site at the anterior proximal aortic arch (Figure 1). The location and size of the entry as well as infectious etiology precluded an endovascular approach, and the anterior entry surrounded by a large amount of thrombi raised significant concern for the intraoperative migration of thrombi into the systemic circulation during the necessary sternal reentry with circulatory arrest.

In the operating room, left femoral artery access was obtained and pre-closed. Cardiopulmonary bypass was initiated via the right axillary artery and femoral vein, with a left ventricular apical vent placed through a small left anterior thoracotomy. When the patient was reaching the goal temperature of $20^{\circ} \mathrm{C}$, a $34 \times 100$ mm Relay graft (Bolton Medical, Sunrise, FL, USA), introduced through the left femoral artery access site, was positioned in the aortic arch under fluoroscopic guidance. Cardiac arrest was then achieved with systemic potassium and deep hypothermic circulatory arrest was induced. The TEVAR graft was promptly deployed to prevent thrombus migration from the PSA (Figure 2)(Figure 3). Occlusion of the orifices of the head vessels with the TEVAR allowed effective antegrade cerebral perfusion via the axillary artery cannula, confirmed by the presence of adequate right radial artery pressure and excellent bilateral cerebral saturations via cerebral oximetry. A repeat median sternotomy immediately followed, upon which the PSA was entered. After thorough debridement of a massive amount of fresh thrombi, a large defect was noted at the prior aortic cannulation site. The innominate artery was dissected out and clamped. The TEVAR graft was explanted through the large entry site. The PSA entry site was extended, and the aortic lumen was carefully inspected to confirm clearance of any potentially embolic particles. The defect was repaired with a bovine pericardial patch, and the systemic circulation was restarted after de-airing. The patient was weaned from bypass without incident and the chest was closed in standard fashion. Cultures from the operating room were positive for MRSA.

He was then taken to the ICU and was extubated within 24 hours. While no postoperative stroke nor recurrence of mediastinitis was observed, the patient suffered a mechanical fall after being successfully discharged from the ICU, and he eventually expired on postoperative day \#75 due to pneumonia.

\section{Comment}

Our case illustrates the novel use of TEVAR to prevent the catastrophic and real potential for embolic stroke on challenging re-entry for aortic PSA repair. This is a rare entity, and previous cardiac surgery is the most common cause of ascending PSA. While endovascular repair has successfully been applied as primary therapy, anastomotic constraints as well as common co-existence of infection may preclude it, requiring open repair. We recognize from prior experience that clots migrating from the PSA into the aorta during the circulatory arrest and sternal opening represent a high embolic risk. To mitigate this risk, we introduced a novel hybrid approach, in which TEVAR was used to prevent embolism of the large volume of hematoma within the pseudoaneurysm. The TEVAR graft accomplished 2 goals: prevention of embolic stroke and maintenance of selective antegrade cerebral perfusion during circulatory arrest.

Although this case represents a unique clinical scenario, requiring an innovative solution, we believe that the interplay between endovascular and open surgical techniques showcased here serves as an excellent example of the potential strategies that could develop and progress the state of the art in aortic surgery and underscores the need for cardiothoracic surgeons to develop a familiarity and skillset with endovascular techniques moving into the future. 


\section{References}

1. Hussain J, Strumpf R, Wheatley G, Diethrich E. Percutaneous closure of aortic pseudoaneurysm by Amplatzer occluder device-case series of six patients.Catheter Cardiovasc Interv. 2009; 73:521-529.

2. Kanani RS, Neilan TG, Palacios IF, Garasic JM. Novel use of the Amplatzer septal occluder device in the percutaneous closure of ascending aortic pseudoaneurysms: a case series.Catheter Cardiovasc Interv. 2007; 69:146-153.

3. Lyen SM, Rodrigues JC, Manghat NE, Hamilton MC, Turner M. Endovascular closure of thoracic aortic pseudoaneurysms: a combined device occlusion and coil embolization technique in patients unsuitable for surgery or stenting.Catheter Cardiovasc Interv. 2016; 88:1155-1169.

4. Kumar PV, Alli O, Bjarnason H, Hagler DJ, Sundt TM, Rihal CS. Percutaneous therapeutic approaches to closure of cardiac pseudoaneurysms. Catheter Cardiovasc Interv. 2012; 80:687-699.

5. F.A. Atik, J.L. Navia, L.G. Svensson, et al. Surgical treatment of pseudoaneurysm of the thoracic aorta. J Thorac Cardiovasc Surg, 132 (2006), pp. 379-385

Figure 1. Preoperative CT angiogram demonstrating a bilobed pseudoaneurysm arising from the ascending aorta, just proximal to the aortic arch takeoff measuring $3.6 \times 2.8 \mathrm{~cm}$ with a large peri-aortic and mediastinal hematoma.

Figure 2. Fluoroscopic image of the TEVAR graft positioned in the proximal aorta

Figure 3. Fluoroscopic image taken after deployment of the TEVAR graft into the ascending aorta.

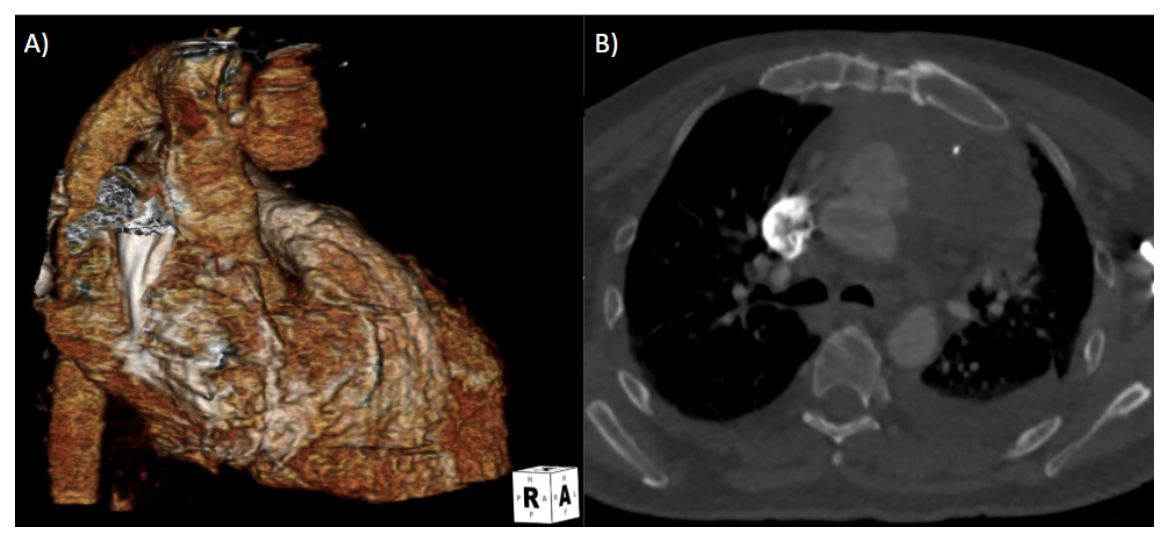




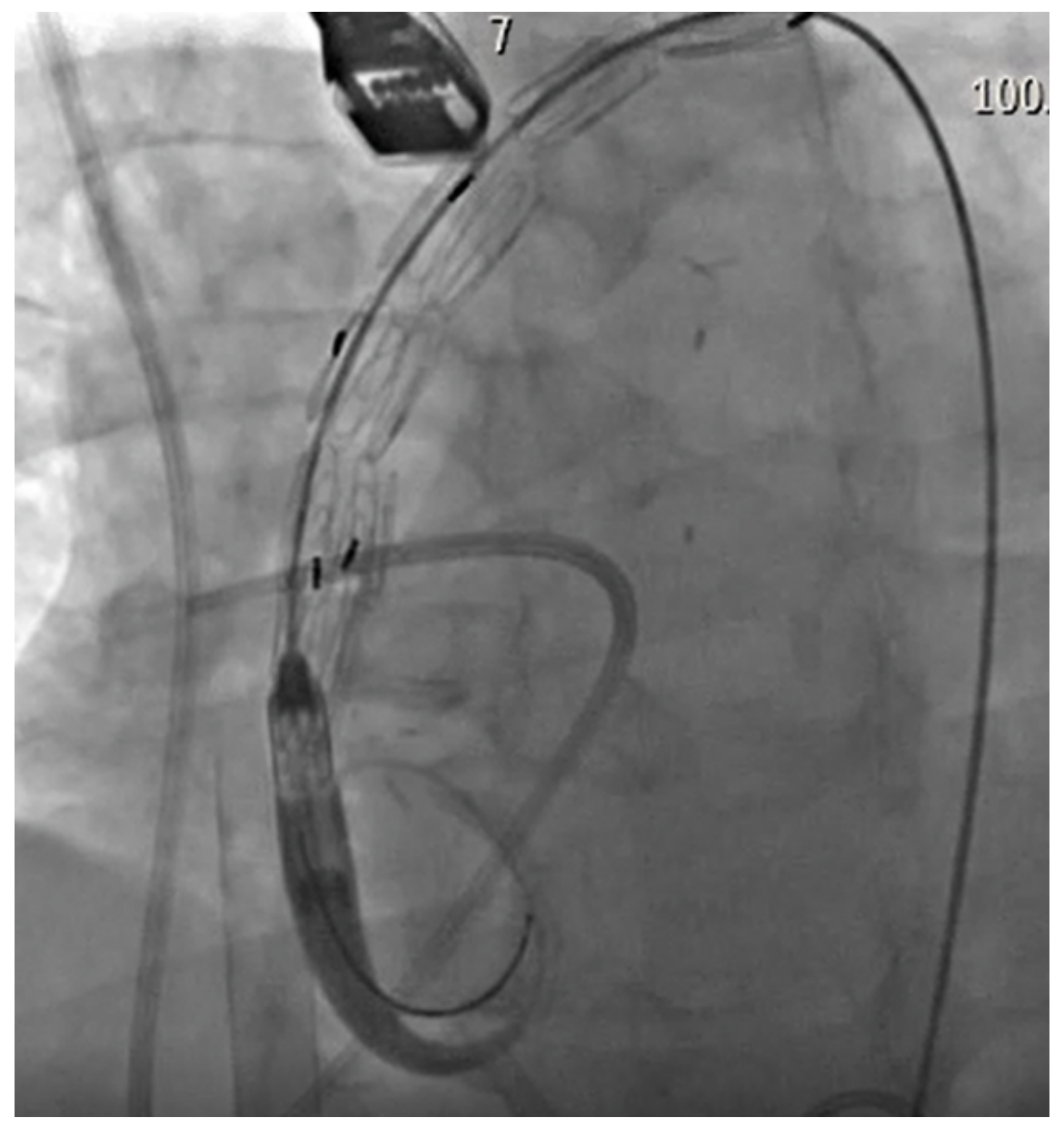




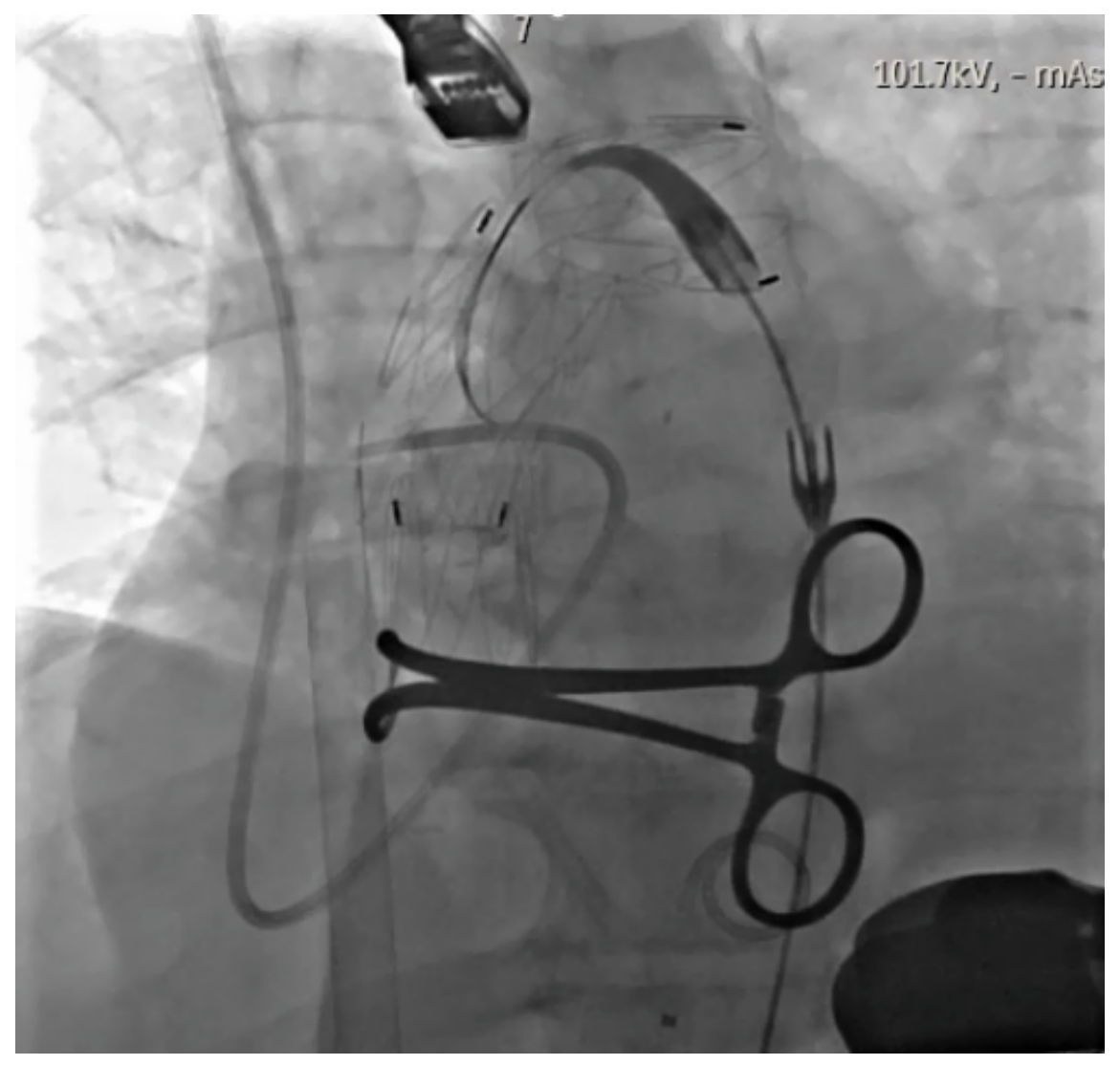

